\title{
Modelo de incremento em área basal para árvores de araucária de uma floresta inequiânea
}

\author{
Emanuel Arnoni Costa ${ }^{1 *}$, César Augusto Guimarães Finger ${ }^{1}$, André Felipe Hess $^{2}$ \\ ${ }^{1}$ Universidade Federal de Santa Maria, Avenida Roraima, n 1.000, CEP 97105-900, Santa Maria, RS, Brasil \\ ${ }^{2}$ Universidade do Estado de Santa Catarina, Av. Luiz de Camões, n 2090, Conta Dinheiro, CEP 88520-000, Lages, SC, Brasil
}

\author{
"Autor correspondente: \\ emanuelarnonicost@hotmail.com \\ Termos para indexação: \\ Araucaria angustifolia \\ Posição sociológica \\ Relações morfométricas \\ Index terms: \\ Araucaria angustifolia \\ Sociological position \\ Morphometric relations
}

\begin{abstract}
Resumo - O presente estudo teve como objetivo desenvolver um modelo matemático para descrever o incremento periódico anual em percentagem para a área basal de árvores de Araucaria angustifolia, com dados coletados de rolos de incrementos extraídos radialmente do tronco de 109 árvores distribuídas em larga amplitude diamétrica. Os atributos dendrométricos e morfométricos foram testados por regressão linear múltipla como variáveis independentes e suas interações com variáveis dummy como descritoras das classes de posição social. O modelo resultante explica $80 \%$ da variação total do incremento periódico anual em área basal percentual com as variáveis formal de copa e a relação altura/diâmetro para árvores de todas as posições sociais, juntamente com o comprimento e proporção de copa das árvores dominantes classificadas por variável dummy. As variáveis morfométricas, principalmente as que caracterizam as dimensões da copa, são indicadas para o desenvolvimento de modelos de crescimento de árvores individuais de araucária.
\end{abstract}

\section{Histórico do artigo:}

Recebido em 06 out 2014

Aprovado em 04 set 2015

Publicado em 30 set 2015

doi: $10.4336 / 2015 . p f b .35 .83 .792$

\section{Basal area increment model of araucaria trees in uneven aged forest}

\begin{abstract}
The present study aimed to develop a mathematical model to describe annual periodic percentage increment in basal area of Araucaria angustifolia trees with data collected from increment cores extracted radially from the trunk at breast height of 109 trees distributed in a large diameter range. Dendrometric and morphometric attributes were tested by multiple linear regressions as independent variables and their interactions with dummy variables as descriptors of social position class. The model explains $80 \%$ of total variation of the periodic annual percentage increment in basal area with formal crown and height/diameter relationship for trees of all social positions, as well as length and crown proportion of dominant trees described by the dummy variable. Morphometric variables, especially those that characterize the crown size, are indicated for development of individual tree growth models of Araucaria angustifolia.
\end{abstract}

\section{Introdução}

O conhecimento de características e relações interdimensionais das árvores pode ser uma alternativa eficaz quando se pretende melhorar a produtividade das florestas, uma vez que vários modelos têm predito o crescimento por meio destas variáveis (Cole \& Lorimer, 1994; Schröder et al., 2002; Cunha et al., 2013).
O intuito de manejar árvores individuais é fornecer condições ideais de crescimento, procurando maximizar o seu incremento. Essas condições podem ser avaliadas pela morfometria das árvores, com reflexo nas condições de espaço vital e de competição, variáveis que possibilitam fazer interferência na dinâmica da floresta.

Os primeiros estudos sobre a dimensão da copa e suas relações se devem a Burger (1939), Assmann (1970), 
Hasenauer \& Monserud (1996) e Hasenauer (1997). No Brasil, alguns autores procuraram descrever a relação de variáveis morfométricas em função de variáveis de fácil medição, como o diâmetro à altura do peito e a altura total, destacando-se os trabalhos de Durlo \& Denardi (1998), Durlo (2001), Tonini \& Arco-Verde (2005), Roman et al. (2009), Padoin \& Finger (2010) e Weber et al. (2013).

Determinar os fatores que influenciam o crescimento refletidos na variação do tamanho e forma da árvore permite definir estratégias adequadas para o planejamento sustentável dos recursos florestais. Essa compreensão também é válida quando associada a diferentes posições sociais na floresta, pois fornece informações adicionais, principalmente quanto ao espaço ideal de crescimento.

Modelos de crescimento em nível de árvore individual possibilitam o controle sobre cada árvore, aplicando-se diretamente tanto em florestas inequiâneas como em povoamentos florestais. Assim, o presente estudo teve por objetivo desenvolver um modelo matemático para descrever o incremento periódico anual em percentagem da área basal de árvores individuais de Araucaria angustifolia (Bertol.) Kuntze em função de variáveis dendrométricas e morfométricas.

\section{Material e métodos}

\section{Área de estudo}

A floresta natural estudada localiza-se em uma propriedade particular com 83,5 ha, no município de Lages, SC ( $27^{\circ} 48^{\prime} \mathrm{S}$ e $\left.50^{\circ} 19^{\prime} \mathrm{O}\right)$. O clima da região é mesotérmico úmido (1.360 a $1.600 \mathrm{~mm}$ de precipitação), sem estação seca definida (Cfb), com temperatura média anual entre $13,8^{\circ} \mathrm{C}$ e $15,8^{\circ} \mathrm{C}$ e umidade relativa de $80 \%$ (Epagri, 2002). Os solos predominantes na região são Nitossolos Háplicos e Cambissolos Húmicos (Sistema..., 1999).

\section{Levantamento dos dados}

Um total de 109 árvores distribuídas nas classes de diâmetro previamente estabelecidas por Hess et al. (2010), foram selecionadas intencionalmente no interior da floresta natural, procurando-se obter um número aproximadamente constante de árvores-amostra em toda a amplitude diamétrica. Mediu-se em cada árvore o diâmetro a 1,30 m do solo (DAP), altura total (h), altura do fuste (hf), oito raios de projeção de copa na direção dos pontos cardeais e colaterais: norte $(\mathrm{N})$, nordeste
(NO), leste (L), sudeste (SE), sul (S), sudoeste (SO), oeste $(\mathrm{O})$ e noroeste $(\mathrm{NO})$ e, por fim, a classificação quanto à posição social (PS). A altura do fuste foi definida como a altura do nível do solo à inserção da copa viva.

$\mathrm{O}$ diâmetro $(\mathrm{cm})$ foi medido com fita diamétrica e a altura $(\mathrm{m})$ e os raios da copa $(\mathrm{cm})$ foram medidos com hipsômetro Vertex IV. O diâmetro de copa (dc) foi determinado duplicando-se o valor médio dos oito raios de copa medidos (média aritmética do raio de copa).

A área de projeção horizontal da copa (APHC) foi calculada por:

$\mathrm{APHC}=\pi$. (média aritmética do raio de copa) ${ }^{2}$.

Em cada árvore foram calculadas as relações morfométricas:

comprimento de copa $=\mathrm{h}-\mathrm{hf}$;

proporção de copa $=\mathrm{cc} / \mathrm{h} * 100$,

formal de copa $=\mathrm{dc} / \mathrm{cc}$,

grau de esbeltez ou relação h/DAP $=$ h/DAP,

índice de abrangência $=\mathrm{dc} / \mathrm{h}$ e

índice de saliência $=\mathrm{dc} / \mathrm{DAP}$.

Onde: $\mathrm{h}=$ altura total; $\mathrm{hf}=$ altura do fuste; $\mathrm{cc}=$ comprimento de copa; dc = diâmetro de copa; DAP = diâmetro a 1,30 $\mathrm{m}$ do solo.

Para a definição da posição social foi considerado o critério silvicultural que avaliou a posição da árvore no estrato vertical da floresta, a presença de árvores vizinhas competidoras e o respectivo grau de exposição da copa à luz. Assim, as seguintes posições sociais foram determinadas: árvore dominante (PS1) - ocupava o estrato superior, com alta exposição da copa à luz; árvore codominante (PS2) - ocupava o estrato intermediário, com média exposição da copa à luz; e árvore dominada (PS3) - posicionada no estrato inferior, com baixa exposição da copa à luz solar (Costa et al., 2014).

\section{Reconstrução do crescimento periódico em área basal}

O crescimento periódico em área basal para um período de cinco anos foi calculado a partir da dimensão dos anéis de crescimento amostrados radialmente ao nível do DAP, utilizando trado de Pressler. Nesse período, considerou-se que as dimensões da copa das árvores não sofreram modificações expressivas, o que é aceito pelo conhecimento da dinâmica neste tipo florestal. Em cada árvore foram coletados dois rolos de incremento, os quais foram acondicionados em canudos de plástico, perfurados e identificados com o número da árvore. 
Em laboratório, fixou-se a amostra com cola em suportes de madeira. Após a secagem, a amostra foi cortada longitudinalmente com estilete, seguindo-se o acabamento da superfície com lixas de diferentes gramaturas, para tornar mais fácil a visualização dos anéis de crescimento.

A medição do incremento foi realizada em mesa micrométrica, com deslocamento horizontal, acoplada a um microscópio estereoscópico. Os dados de incremento foram registrados pelo programa Time Series Analysis Program - TSAP (Rinn, 2003) e o incremento periódico anual em área basal foi expresso na forma relativa por:

$$
\mathrm{IPAg} \%=\left(\frac{g-g_{5}}{g_{5}}\right) \cdot\left(\frac{100}{n}\right)
$$

Em que: $\mathrm{IPAg} \%=$ Incremento periódico anual em percentagem da área basal para um período de 5 anos; $\mathrm{g}=$ área basal no final do período, $\mathrm{em}^{2} ; \mathrm{g}_{5}=$ área basal no início do período; em $\mathrm{cm}^{2} ; \mathrm{n}=$ número de anos avaliados.

\section{Modelagem e avaliação do modelo de incremento periódico em área basal}

O efeito da heterocedasticidade e não normalidade dos dados foi contornado com a transformação da variável dependente utilizando a família de transformações BoxCox (Box \& Cox, 1964). A modelagem do ln (IPAg\%) foi realizada pelo procedimento Stepwise no Sistema de Análise Estatística - SAS V. 9.1 (Sas Institute, 2004), em função das variáveis dendrométricas e morfométricas mais correlacionadas, juntamente com a interação de variáveis discretas dummy designadas pelas classes de posições sociais que as árvores ocupavam (dummy D1 - PS1; D2 - PS2 e D3 - PS3).

$\mathrm{Na}$ avaliação do modelo de regressão os resíduos foram avaliados com os testes de White para a homogeneidade; teste Shapiro-Wilk, para a normalidade; e teste de Durbin-Watson, para a independência. A distância de Cook's $\left(D_{i}\right)$ foi empregada na determinação de observações influentes, "outliers", sendo que observações afastadas da massa de pontos ( $\mathrm{Di}>1)$ foram identificadas, examinadas na base de dados e, quando necessário, eliminadas (Weisberg, 2005). Nos casos em que o modelo foi reajustado sem esses pontos e apresentou melhoria no ajuste, os pontos foram considerados influentes e eliminados da base de dados.

A colinearidade na estimativa dos coeficientes de regressão foi examinada com base no fator de inflação da variância (FIV), sendo aceitos valores menores que 10 como ausência de multicolinearidade (Kutner et al., 2004). A avaliação do ajuste e precisão do modelo foi baseada no coeficiente de determinação ajustado ( $\left.R^{2} a j.\right)$, no erro padrão da estimativa em percentagem (Syx\%) e na distribuição gráfica dos resíduos.

\section{Resultados e discussão}

A transformação de Box \& Cox (1964) apresentou valor de lambda igual a zero $(\lambda=0)$, indicando a transformação logarítmica $(\ln )$ para reduzir a variância da variável dependente (IPAg\%).

A modelagem do $\ln (\mathrm{IPAg} \%)$ foi realizada por meio da seleção Stepwise em função de atributos dendrométricos e morfométricos das árvores mais correlacionados (Tabela 1), e a interação com variáveis de classe descritoras das posições sociais (variáveis dummy).

Tabela 1. Correlação de Pearson e a probabilidade em função de variáveis dendrométricas e morfométricas de araucária em floresta natural.

\begin{tabular}{lc}
\hline \multicolumn{1}{c}{ Variáveis } & $\begin{array}{c}\text { Correlação de Pearson } \\
\text { entre In (IPAg\%) e } \\
\text { variáveis }\end{array}$ \\
\hline Diâmetro a 1,30 m do solo $(\mathrm{cm})$ & $-0,68^{*}$ \\
Altura $(\mathrm{m})$ & $-0,63^{*}$ \\
Altura do fuste $(\mathrm{m})$ & $-0,73^{*}$ \\
$\begin{array}{l}\text { Diâmetro de copa }(\mathrm{m}) \\
\text { Área de projeção horizontal da copa } \\
\text { (m²) }\end{array}$ & $-0,62^{*}$ \\
Comprimento de copa (m) & $-0,60^{*}$ \\
Proporção de copa $(\%)$ & $0,34^{*}$ \\
Formal de copa & $0,67^{*}$ \\
Relação altura/DAP & $-0,75^{*}$ \\
Índice de saliência & $0,48^{*}$ \\
\hline
\end{tabular}

${ }^{*} \mathrm{P}<0,0001$.

No processo de seleção (Tabela 2), foram adicionadas as variáveis formal de copa $(\mathrm{Fc})$, seguidas das variáveis proporção de copa das árvores dominantes (Pc.D1), comprimento de copa das árvores dominantes (cc.D1), e a variável de relação $\mathrm{h} / \mathrm{DAP}$, que originaram a regressão: 


\section{$\ln (\mathrm{IPAg} \%)=$ função $(\mathrm{Fc} ; \mathrm{cc} . \mathrm{D} 1 ; \mathrm{Pc} . \mathrm{D} 1 ; \mathrm{h} / \mathrm{DAP})$}

Em que: $\ln (\mathrm{IPAg} \%)=$ logaritmo natural do incremento periódico anual em percentagem da área basal; $\mathrm{Fc}=$ formal de copa; cc.D1 = comprimento de copa de árvores dominantes (D1 - Dummy para árvores dominantes PS1); Pc. D1 = proporção de copa de árvores dominantes (D1 - Dummy para árvores dominantes - PS1); h/DAP $=$ relação $\mathrm{h} / \mathrm{DAP}$.

Tabela 2. Resumo estatístico da seleção stepwise de árvores de araucária em floresta natural.

\begin{tabular}{ccccc}
\hline Variável & $\mathbf{R}_{\text {parcial }}^{\mathbf{2}}$ & $\mathbf{R}_{\text {equacão }}^{\mathbf{2}}$ & $\mathbf{F}$ & $\mathbf{P}>\mathbf{F}$ \\
\hline Fc & 0,5587 & 0,5587 & 131,69 & $<0,0001$ \\
Pc.D1 & 0,0465 & 0,6053 & 12,14 & 0,0007 \\
cc.D1 & 0,1568 & 0,7621 & 67,23 & $<0,0001$ \\
h/DAP & 0,0492 & 0,8113 & 26,34 & $<0,0001$ \\
\hline
\end{tabular}

Em que: $\mathrm{R}_{\text {parcial }}^{2}=$ coeficiente de determinação parcial; $\mathrm{R}_{\text {equação }}^{2}=$ coeficiente de determinação da equação; $\mathrm{F}=$ valor da estimativa de $\mathrm{F} ; \mathrm{Fc}=$ formal de copa; $\mathrm{Pc} . \mathrm{D} 1$ = proporção de copa das árvores dominantes, cc.D1 = comprimento de copa das árvores dominantes; $\mathrm{h} / \mathrm{DAP}=$ relação h/DAP

A maior parte da variação explicada pelo modelo de regressão $(\approx 56,0 \%)$ esteve associada às características de dimensão da copa $(\mathrm{Fc})$ nas árvores de todas as posições sociais seguida da proporção e comprimento de copa $(\approx 20,0 \%)$ nas árvores da classe dominante (Pc.D1 e cc.D1), respectivamente. Esse resultado permite confirmar a condição biológica da espécie em que árvores com maior proporção de copa apresentam maior crescimento, comparado com árvores de posição social codominante e dominada. A relação h/DAP foi a variável de menor contribuição, considerando todas as classes sociais ocupadas pelas árvores (Tabela 2).

Características de dimensão da árvore e da copa, em especial, também foram empregadas para modelar o incremento em área basal de árvores individuais de Cedrela odorata L., na Floresta Amazônica, com base nos atributos de h, relação h/d e Fc (Cunha et al., 2013), onde a maior parte da variação foi descrita pelos atributos associados à copa, indicando ser este um referencial para a predição do crescimento quando se deseja maior precisão e confiança dos modelos matemáticos. Também, Monserud \& Sterba (1996) desenvolveram um modelo de incremento em área basal de árvores individuais utilizando variáveis de tamanho, competição e de sítio. As variáveis que descreviam o tamanho (DAP e cc) explicaram, de acordo com a espécie, entre $14,1 \%$ e $47,2 \%$ da variação do incremento em área basal.
A dimensão da copa e a posição social representam o efeito de competição a que a árvore esteve sujeita no passado, e que atuou como regulador do tamanho e da forma da copa, bem como da taxa de crescimento. Esse efeito determinou a maior redução do comprimento e diâmetro de copa (cc e dc), refletindo na maior redução do incremento de árvores dominadas e codominantes, quando comparadas com árvores dominantes. A relação entre as condições de crescimento no passado e o cc e demais dimensões da árvore também foram abordadas por Hasenauer \& Monserud (1996).

Assmann (1970), por sua vez, já considerava a morfometria da copa como uma importante medida de vigor da árvore, sendo copas estreitas e longas as que possibilitam maior crescimento, permitindo que maior número de árvores cresça por unidade de área.

$\mathrm{Na}$ modelagem do $\ln (\mathrm{IPAg} \%)$ o intercepto não foi significante a 5\%. Entretanto, foi mantido ao se verificar que sua retirada do modelo não trouxe ganho na performance preditiva (Tabela 3 ). As variáveis independentes incluídas no modelo tiveram fator de inflação de variância (FIV) menor que 6,5, indicando ausência de inflação na variância.

Tabela 3. Análise de variância e parâmetros estimados do modelo logarítmico de incremento periódico anual em percentagem da área basal (IPAg\%) de araucária em floresta natural

\begin{tabular}{cccccc}
\hline FV & GL & SQ & QM & $\mathbf{F}$ & $\mathbf{p}>\mathbf{F}$ \\
\hline Modelo & 4 & 75,25719 & 18,81430 & 108,57 & $<0,0001$ \\
Erro & 101 & 17,50262 & 0,17329 & - & - \\
Total & 105 & 92,75981 & - & - & - \\
\hline Variável & Parâmetros & Erro padrão & Valor de t & Pr. $>|\mathbf{t}|$ & FIV \\
\hline Intercepto & $-0,3235$ & 0,35700 & $-0,91$ & $<0,3670$ & 0 \\
Fc & $-0,2087$ & 0,05395 & $-3,87$ & $<0,0002$ & 2,6 \\
h/DAP & 2,0878 & 0,40680 & 5,13 & $<0,0001$ & 2,4 \\
cc.D1 & $-0,2482$ & 0,03780 & $-6,57$ & $<0,0001$ & 6,5 \\
Pc.D1 & 0,0586 & 0,00556 & 10,53 & $<0,0001$ & 6,5 \\
\hline
\end{tabular}

Em que: $\mathrm{FV}=$ fonte de variação; $\mathrm{GL}=$ graus de liberdade; $\mathrm{SQ}=$ soma de quadrados; $\mathrm{QM}=$ quadrado médio; $\mathrm{F}=$ valor de $\mathrm{F} ; \mathrm{p}>\mathrm{F}=$ probabilidade de significância para o valor de F; FIV= fator de inflação de variância; $\mathrm{Fc}=$ formal de copa; Pc.D1 = proporção de copa das árvores dominantes, em \%, cc.D1 = comprimento de copa das árvores dominantes, em m; h/DAP = relação h/DAP.

A distância de Cook utilizada para o diagnóstico de observações influentes identificou três pontos, que foram verificados e eliminados da base de dados, proporcionando aumento significativo de todos os estimadores das variáveis independentes. 
As predições dos $\ln (\mathrm{IPAg} \%)$ geradas com o modelo apresentam maior acurácia e dispersão uniforme dos valores em toda a amplitude diamétrica (Figura 1), com mais de $80,0 \%$ da variação do ln (IPAg\%) explicado pela regressão $\left(\mathrm{R}^{2}{ }_{\text {aj }}\right)$ e com erro padrão da estimativa em porcentagem $\left(\mathrm{S}_{\mathrm{yx}} \%\right)$ de $25,77 \%$.

Em região ecologicamente semelhante à de origem dos dados deste trabalho, Zanon (2007) desenvolveu um modelo de incremento em área basal para araucária com base em variáveis meteorológicas, morfométricas e ambientais, tendo o modelo resultante explicado $84,1 \%$ $\left(\mathrm{R}_{\text {ai }}^{2}\right)$ da variação total.
A contribuição das variáveis independentes no modelo de regressão (Figura 2a), mostrou o decréscimo do $\ln$ (IPAg\%) à medida que o formal de copa $(\mathrm{Fc})$ aumentava, indicando que árvores com copa curta e larga tiveram menor incremento quando comparadas com árvores de copa longa e estreita (menor $\mathrm{Fc}$ ). O resultado comprovou que as árvores de melhor posição social (PS1) são mais eficientes se comparadas com as codominantes (PS2) e as dominadas (PS3). Valores da relação h/DAP entre 0,35 e 0,62 , encontrados para árvores jovens com DAP pequeno e alta proporção de copa, representadas na parte superior da Figura 2b, apresentaram maior incremento.

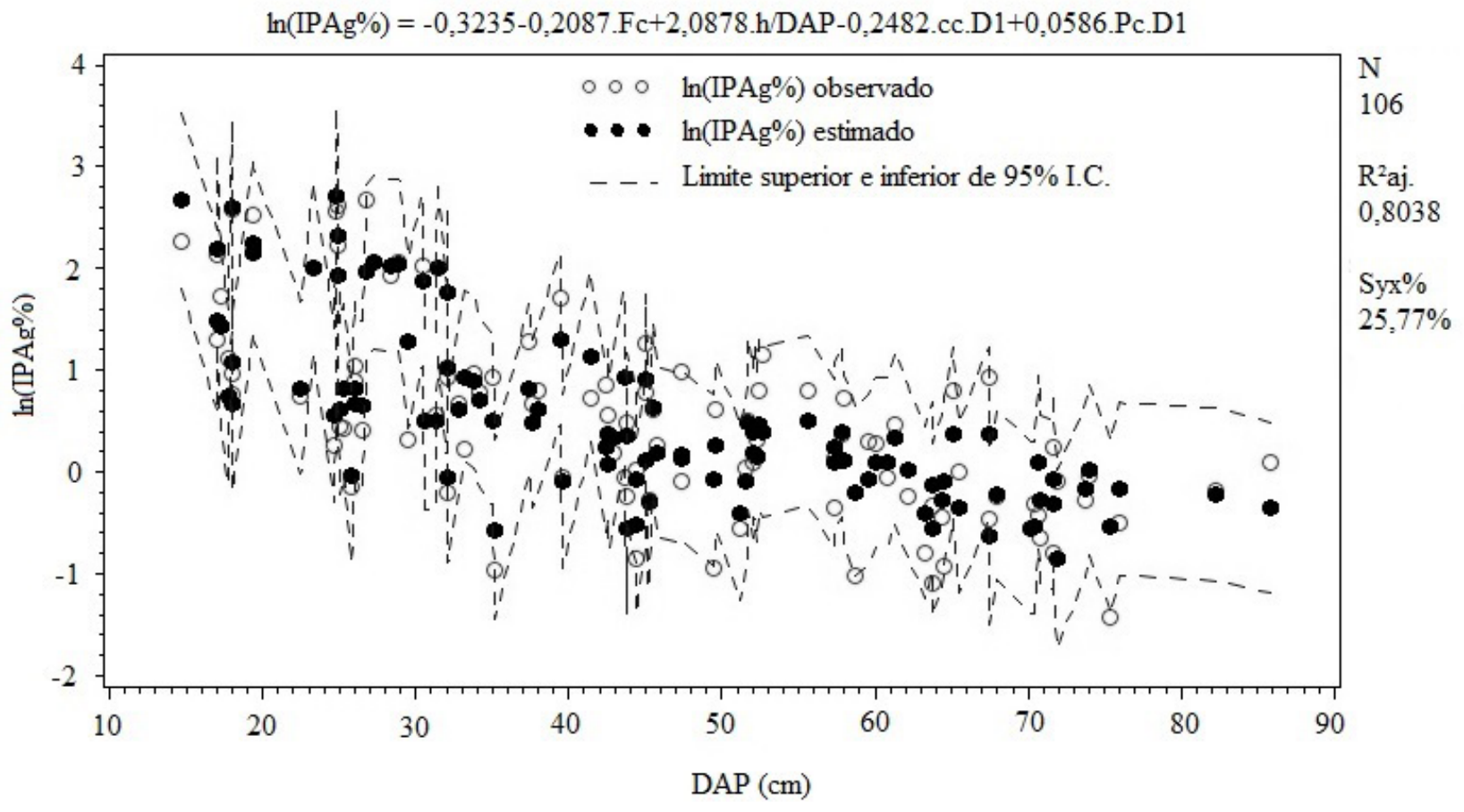

Figura 1. Distribuição do logaritmo do incremento periódico anual em área basal percentual - $\ln (\operatorname{IPAg} \%)$ observado e ajustado em função do diâmetro a 1,30 m do solo, em árvores individuais de araucária em floresta natural.

As variáveis comprimento e proporção de copa (cc e Pc, respectivamente) foram significantes apenas para as árvores dominantes (Dummy D1 - PS1), mostrando o aumento crescente do incremento à medida que aumenta o comprimento de copa e a proporção de copa. Na Figura $2 \mathrm{c}$ e $2 \mathrm{~d}$ os pontos indicados com valor zero na abscissa referem-se às árvores codominantes (PS2) e dominadas (PS3), cuja variável dummy não foi significante e, por essa razão, apareceram nesta posição na figura.

A dispersão dos resíduos padronizados em função do incremento periódico em área basal (Figura 3) revelou existir cinco pontos além dos limites (dentro da área de rejeição), a considerar o valor crítico do $t$ de Student $\mathrm{em} \pm 1,98$ para 105 graus de liberdade. Os pontos fora destes limites foram analisados na base de dados e não tendo sido associados a qualquer erro, foram mantidos, pois estatisticamente eram admissíveis.

Os testes de condicionantes de regressão indicaram resíduos homocedásticos pelo teste de White $\left(\mathrm{p}>\mathrm{x}^{2}=0,4092\right)$, com distribuição normal pelo teste de Shapiro Wilk $(\mathrm{p}=0,2703)$. Além destes, o teste de Durbin-Watson revelou não haver indícios de correlação entre erros $(\mathrm{DW}=1,8)$ positivos $(\mathrm{p}<\mathrm{DW}=0,1155) \mathrm{e}$ negativos $(\mathrm{p}>\mathrm{DW}=0,8845)$, comprovando um bom ajuste e precisão do modelo. 


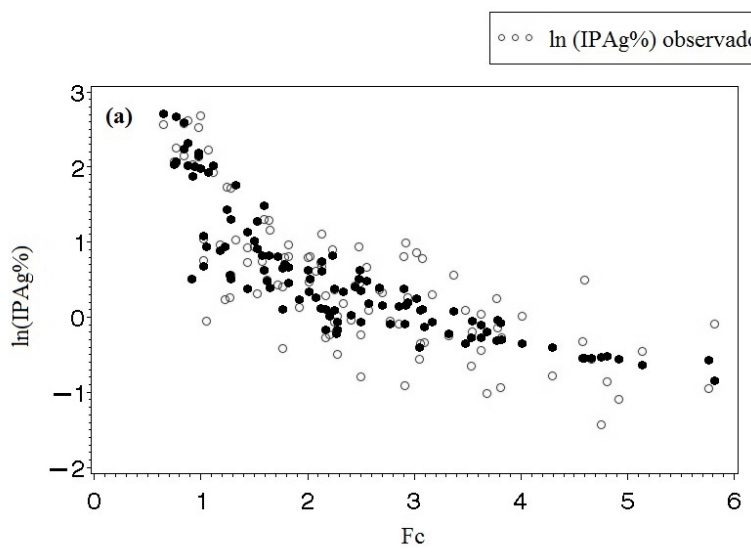

$\cdots \ln (\mathrm{IPAg} \%)$ estimado
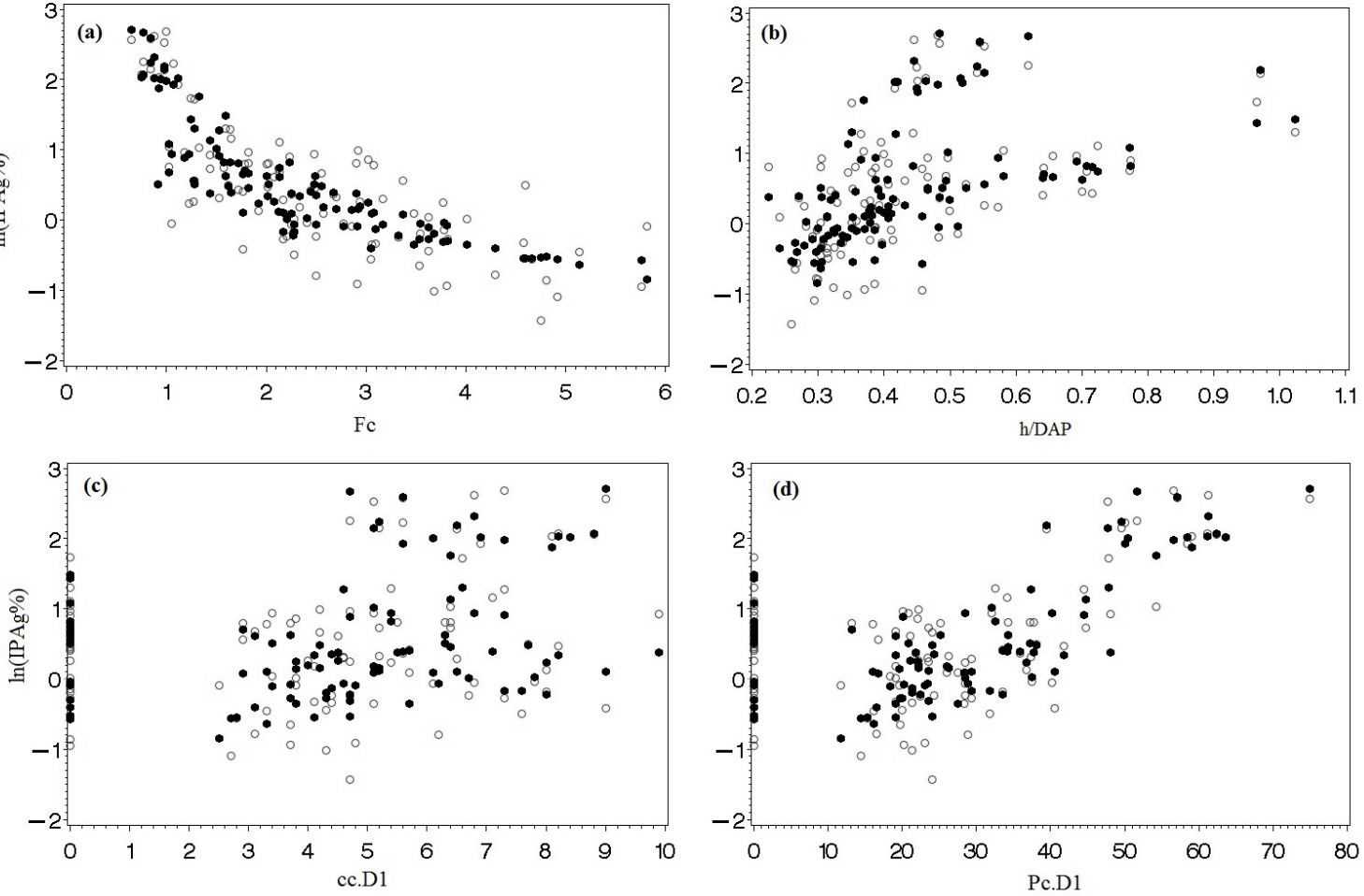

Figura 2. Distribuição de valores estimados e observados do modelo logarítmico de incremento periódico anual em percentagem da área basal (ln (IPAg\%)) em função de cada variável independente de árvores de araucária em floresta natural: a) formal de copa $(\mathrm{Fc})$; b) relação h/DAP; c) comprimento de copa para árvores dominantes (cc.D1); d) proporção de copa para árvores dominantes (Pc.D1).

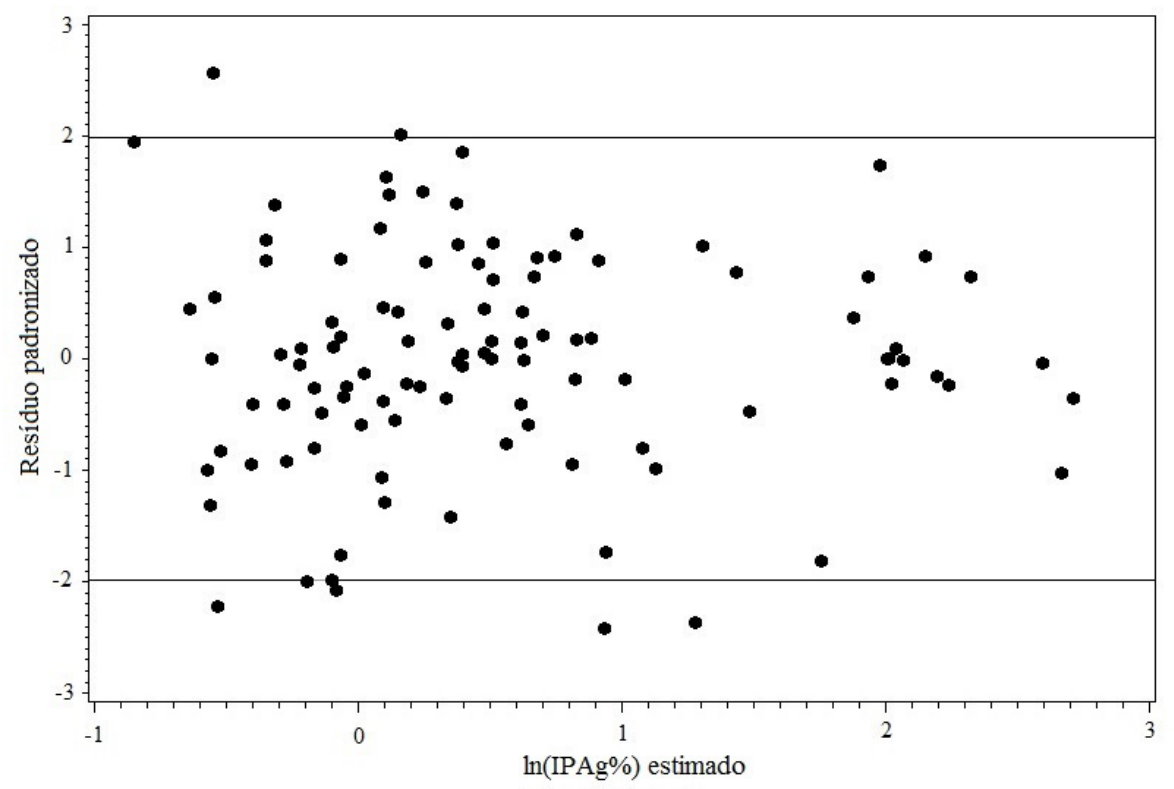

Figura 3. Resíduo padronizado em função $\ln (\mathrm{IPAg} \%)$ estimado para árvores de araucária em floresta natural. 


\section{Conclusões}

$\mathrm{O}$ incremento periódico anual em percentagem da área basal pode ser estimado com precisão, tendo como variáveis independentes o formal de copa e a relação h/ DAP das árvores, incluindo, além dessas, as variáveis comprimento e proporção de copa para as árvores dominantes.

As variáveis morfométricas, principalmente as que caracterizam as dimensões da copa, são indicadas para o desenvolvimento de modelos de crescimento de árvores de araucária.

\section{Referências}

ASSMANN, E. The principles of forest yield study. Oxford: Pergamon, 1970. $506 \mathrm{p}$.

BOX, G. E. P.; COX, D. R. An analysis of transformations. Journal of the Royal Statistical Society, Series B (Methodological), London, v. 26, n. 2, p. 211-243, 1964.

BURGER, H. B. Zuwachs in zwei hiebsreifen Fichtenbeständen. Mitteilungen der Schweizerischen Anstalt Für Forstliche Versuchswesen, Zürich, v. 21, p. 147-176, 1939.

COLE, W. G.; LORIMER, C. G. Predicting tree growth from crown variables in managed northern hardwood stands. Forest Ecology and Management, Amsterdam, v. 67, p. 159-175, 1994. DOI: 10.1016/0378-1127(94)90014-0

COSTA, E. A.; FINGER, C. A. G.; CUNHA, T. A. Influência da posição sociológica na relação hipsométrica de Araucaria angustifolia. Revista Brasileira de Ciências Agrárias, Recife, v. 9, n. 1, p. 110-116, 2014. DOI:10.5039/agraria.v9i1a3201

CUNHA, T. A.; FINGER, C. A. G.; SCHNEIDER, P. R. Linear mixed model to describe the basal area increment for individual cedro (Cedrela odorata L.) trees in Occidental Amazon, Brazil. Ciência Florestal, Santa Maria, RS, v. 23, n. 3, p. 461-470, 2013. DOI: $10.5902 / 1980509810557$

DURLO, M. A.; DENARDI, L. Morfometria de Cabralea canjerana, em mata secundária nativa do Rio Grande do Sul. Ciência Florestal, Santa Maria, RS, v. 8, n. 1, p. 55-66, 1998.

DURLO, M. A. Relações morfométricas para Cabralea canjerana (Well.) Mart. Ciência Florestal, Santa Maria, RS, v. 11, n. 1, p. 141-150, 2001.

EPAGRI. Dados e informações biofísicas da Unidade de Planejamento Regional Planalto Sul Catarinense: UPR 3. Florianópolis, 2002. 70 p.
HASENAUER, H. Dimensional relationships of open-grown trees in Austria. Forest Ecology and Management, Amsterdam, v. 96, p. 197-206, 1997.

HASENAUER, H.; MONSERUD, R. A. A crown ratio model for Austrian forests. Forest Ecology and Management, Amsterdam, v. 84, p. 49-60, 1996.

HESS, A. F.; CALGAROTTO, A. R.; PINHEIRO, R.; WANGINIAK, T. C. R. Proposta de manejo de Araucaria angustifolia (Bertol.) Kuntze utilizando o quociente de Liocourt e análise de incremento, em propriendade rural no Município de Lages, SC. Pesquisa Florestal Brasileira, Colombo, v. 30, n. 64, p. 337-345, 2010. DOI: 10.4336/2010.pfb.30.64.337

KUTNER, M. H.; NACHTSHEIM, C. J.; NETTER, J.; LI, W. Applied linear statistical models. 5. ed. Boston: McGraw-Hill Irwin, 2004. $1396 \mathrm{p}$.

MONSERUD, R. A.; STERBA, H. A basal area increment model for individual trees growing in even- and uneven-aged forest stands in Austria. Forest Ecology and Management, Amsterdam, v. 80, p. 57-80, 1996.

PADOIN, V.; FINGER, C. A. G. Relações entre as dimensões da copa e a altura das árvores dominantes em povoamentos de Pinus taeda L. Ciência Florestal, Santa Maria, RS, v. 20, n. 1, p. 95-105, 2010.

RINN, F. TSAP-Win: version 4.64, reference manual: time series analysis and presentation dendrochronology and related applications. Heidelberg, 2003. 110 p.

ROMAN, M.; BRESSAN, D. A.; DURLO, M. A. Variáveis morfométricas e relações interdimensionais para Cordia trichotoma (Vell.) Arráb. Ex Steud. Ciência Florestal, Santa Maria, RS, v. 19, n. 4, p. 473-480, 2009.

SAS Institute. The SAS system for Windows. Cary, 2004.

SCHRÖDER, J.; SOALLEIRO, R. R.; ALONSO, G. V. An ageindependent basal area increment model for maritime pine trees in northwestern Spain. Forest Ecology and Management, Amsterdam, v. 157, p. 55-64, 2002.

SISTEMA Brasileiro de classificação de solos. Brasília, DF: Embrapa Produção de Informação; Rio de Janeiro: Embrapa Solos, 1999. $412 \mathrm{p}$.

TONINI, H.; ARCO-VERDE, M. F. Morfologia da copa para avaliar o espaço vital de quatro espécies nativas da Amazônia. Pesquisa Agropecuária Brasileira, Brasília, DF, v. 40, n. 7, p. 633-638, 2005.

WEBER, V. P.; FINGER, C. A. G.; FLEIG, F. D.; SOUZA, C. A. M.; MUNARETO, F. F.; SILVA, L. T. M. Diretrizes para desbaste de Pinus taeda L. em função da altura dominante. Ciência Florestal, Santa Maria, RS, v. 23, n. 1, p. 193-201, 2013.

WEISBERG, S. Applied linear regression. 3. ed. New York: Wiley, 2005. 310 p.

ZANON, M. L. B. Crescimento da Araucaria angustifolia (Bertol.) kuntze diferenciado por dioicia. 2007. 110 f. Tese (Doutorado em Ciências Florestais) - 
